\title{
Review Article \\ Green Nanobiotechnology: Factors Affecting Synthesis and Characterization Techniques
}

\author{
Jayanta Kumar Patra and Kwang-Hyun Baek \\ School of Biotechnology, Yeungnam University, Gyeongsan, Gyeongbuk 712-749, Republic of Korea \\ Correspondence should be addressed to Kwang-Hyun Baek; khbaek@ynu.ac.kr
}

Received 13 October 2014; Accepted 1 December 2014; Published 17 December 2014

Academic Editor: Wei Chen

Copyright (C) 2014 J. K. Patra and K.-H. Baek. This is an open access article distributed under the Creative Commons Attribution License, which permits unrestricted use, distribution, and reproduction in any medium, provided the original work is properly cited.

Nanobiotechnology is gaining tremendous impetus in this era owing to its ability to modulate metals into their nanosize, which efficiently changes their chemical, physical, and optical properties. Accordingly, considerable attention is being given to the development of novel strategies for the synthesis of different kinds of nanoparticles of specific composition and size using biological sources. However, most of the currently available techniques are expensive, environmentally harmful, and inefficient with respect to materials and energy use. Several factors such as the method used for synthesis, $\mathrm{pH}$, temperature, pressure, time, particle size, pore size, environment, and proximity greatly influence the quality and quantity of the synthesized nanoparticles and their characterization and applications. Additionally, characterization of the synthesized nanoparticles is essential to their potential use in various drug delivery and biomedical applications. The present review highlights various parameters affecting the synthesis of nanoparticles by green nanobiotechnology and different techniques used for characterizing the nanoparticles for their potential use in biomedical and environmental applications.

\section{Introduction}

The synthesis of nanoparticles using modern techniques has emerged as an important application in the biomedical and human health care field for a broad range of products [1]. In general, nanotechnology can be defined as manipulation of materials at the atomic level by a combination of engineering, chemical, and biological approaches [2]. The modern concept of nanotechnology which is now considered to be the most widely studied field in science has a history dating back to the ninth century, when nanoparticles of gold and silver were used by the artisans of Mesopotamia age to generate a glittering effect on pots and other utensils [3]. However, the first scientific description of nanoparticle properties was provided by Michael Faraday in his famous paper "Experimental Relations of Gold and Other Metals to Light" [3, 4]. The term "nanotechnology" was used for the first time by Richard Feynman in 1959, which is considered to mark the beginning of modern nanotechnology [4].

Numerous efforts are being made throughout the world to develop ecofriendly technologies to produce environmentally benign, nontoxic products using green nanotechnology and biotechnological tools $[5,6]$. Nanoparticles synthesized using biological methods or green technology have diverse natures, with greater stability and appropriate dimensions since they are synthesized using a one-step procedure. Various undesirable processing conditions are thus eliminated by allowing the synthesis to proceed at physiological temperatures, $\mathrm{pH}$, pressure, and, at the same time, a negligible cost [7]. Thus, specific characterization techniques may be employed to characterize the potential for application of synthesized nanoparticles for use in drug delivery and biomedical fields.

To date, no detailed investigations of the factors affecting the synthesis of various types of nanoparticles using green technology, their characterization, or their analysis have been published. Therefore, this review was conducted to highlight the factors influencing nanoparticle synthesis using green technology and different techniques for characterization of nanoparticles to provide a better understanding of nanoparticles and thus improve their uses in modern technology. 


\section{Green Nanotechnology}

Nanoparticles can be synthesized using a variety of methods including physical, chemical, biological, and hybrid techniques (Figure 1) [8-10]. The production of nanoparticles through conventional physical and chemical methods results in toxic byproducts that are environmental hazards. Additionally, these particles cannot be used in medicine due to health-related issues, especially in clinical fields $[11,12]$. Conventional methods can be used to produce nanoparticles in large quantities with defined sizes and shapes in a shorter period of time; however, these techniques are complicated, costly, inefficient, and outdated. In recent years, there has been growing interest in the synthesis of environmentally friendly nanoparticles that do not produce toxic waste products during the manufacturing process $[6,13,14]$. This can only be achieved through benign synthesis procedures of a biological nature using biotechnological tools that are considered safe and ecologically sound for nanomaterial fabrication as an alternative to conventional physical and chemical methods [5]. This has given rise to the concept of green technology or green nanobiotechnology.

In general, green nanobiotechnology means synthesizing nanoparticles or nanomaterials using biological routes such as those involving microorganisms, plants, and viruses or their byproducts, such as proteins and lipids, with the help of various biotechnological tools. Nanoparticles produced by green technology are far superior to those manufactured with physical and chemical methods based on several aspects. For example, green techniques eliminate the use of expensive chemicals, consume less energy, and generate environmentally benign products and byproducts. The 12 principles of green chemistry have now become a reference guide for researchers, scientists, chemical technologists, and chemists around the world for developing less hazardous chemical products and byproducts $[15,16]$. Accordingly, green nanobiotechnology is a promising alternate route for synthesis of biocompatible stable nanoparticles [17].

Biological-based synthesis of nanoparticles utilizes a bottom-up approach in which synthesis occurs with the help of reducing and stabilizing agents (Figure 2). Three main steps are followed for the synthesis of nanoparticles using a biological system: the choice of solvent medium used, the choice of an ecofriendly and environmentally benign reducing agent, and the choice of a nontoxic material as a capping agent to stabilize the synthesized nanoparticles $[4,8]$. Nanotechnology has more advantages over other conventional approaches owing to the availability of more components by biological system for the formation of nanoparticles. The rich biodiversity of such biological components has been explored for the synthesis of bionanomaterials, which are environmentally benign and can be used in various medical applications.

\section{Factors Affecting the Synthesis of Green Nanoparticles}

There are several factors that affect the synthesis, characterization, and application of nanoparticles. Many researchers

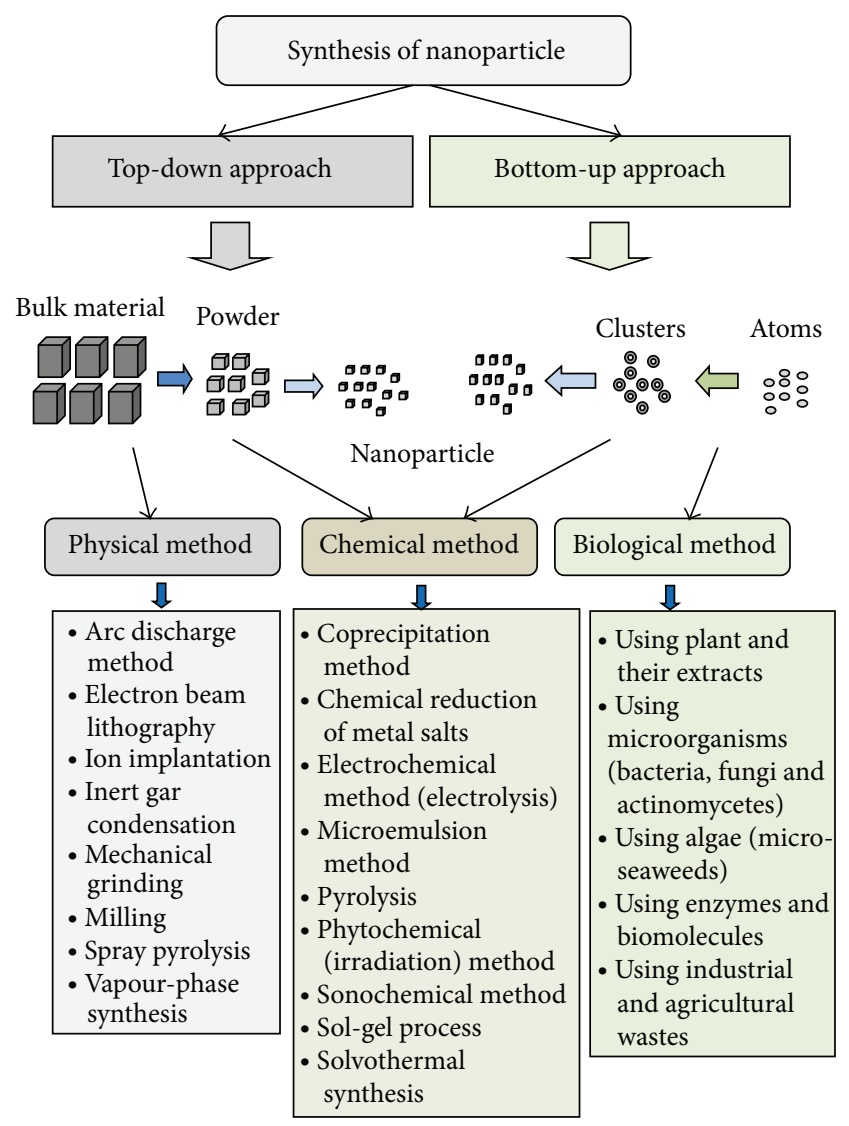

FIgURE 1: Different approaches and methods for synthesizing nanoparticles.

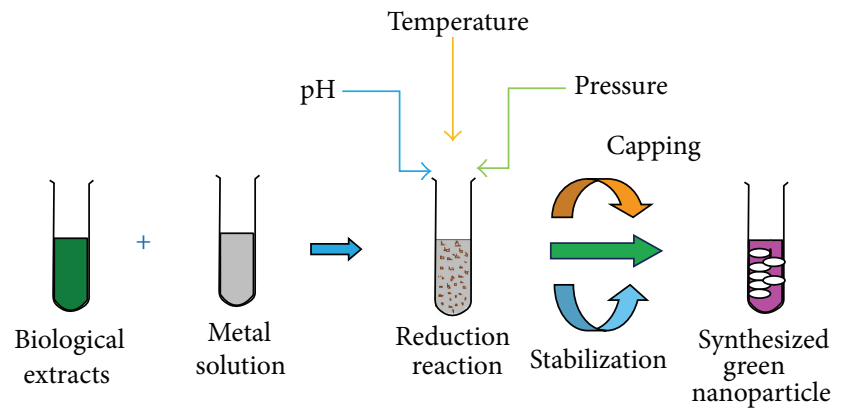

FIGURE 2: Biological synthesis of nanoparticles using green technology.

have reported the change in nature of the synthesized nanoparticles with the type of the adsorbate and the activity of the catalysts used in the synthesis process $[18,19]$. Some of them have reported the dynamic nature of the synthesized nanoparticles with different types of symptoms and implications by change with time and environment, and so forth [20]. There are several other important factors that affect the synthesis of nanoparticles, including $\mathrm{pH}$ of the solution, temperature, concentration of the extracts used, concentration of the raw materials used, size, and above all the protocols that are followed for the synthesis process [21]. 
Some dominant factors that affect nanoparticle biosynthesis are described below.

3.1. Particular Method or Technique. There are different methods for synthesizing nanoparticles, ranging from physical techniques using mechanical procedures to chemical or biological protocols using various organic or inorganic chemicals and living organisms. Each procedure has specific benefits and drawbacks. However, biological methods for synthesis of nanoparticles use nontoxic and environmentally benign materials in conjunction with green technology and are therefore eco-friendly and more acceptable than traditional methods $[16,22]$.

3.2. $p H . \mathrm{pH}$ is an important factor that influences the synthesis of nanoparticles by green technology methods. Researchers have discovered that $\mathrm{pH}$ of the solution medium influences the size and texture of the synthesized nanoparticle $[23,24]$. Therefore, nanoparticle size can be controlled by altering the $\mathrm{pH}$ of the solution media. The effect of $\mathrm{pH}$ on the shape and size of the synthesized silver nanoparticle was demonstrated by Soni and Prakash [25].

3.3. Temperature. Temperature is another important parameter that affects the synthesis of nanoparticles using all three methods. The physical method requires the highest temperature $\left(>350^{\circ} \mathrm{C}\right)$, whereas chemical methods require a temperature less than $350^{\circ} \mathrm{C}$. In most cases, the synthesis of nanoparticles using green technology requires temperatures less than $100^{\circ} \mathrm{C}$ or ambient temperature. The temperature of the reaction medium determines the nature of the nanoparticle formed [26].

3.4. Pressure. Pressure is important for the synthesis of nanoparticles. The pressure applied to the reaction medium affects the shape and size of the synthesized nanoparticles [27]. The rate of reduction of metal ions using biological agents has been found to be much faster at ambient pressure conditions [28].

3.5. Time. The quality and type of nanoparticle synthesized using green technology are greatly influenced by length of time for which the reaction medium is incubated [29]. Similarly the characteristics of the synthesized nanoparticles were also altered with time and greatly influenced by the synthesis process, exposure to light, and storage conditions, and so forth $[30,31]$. The variations in the time may occur in many ways such as aggregation of particles due to long time storage; particles may shrink or grow during long storage; they may have shelf life, and so forth, that affects their potential [32].

3.6. Preparation Cost. To facilitate the potential application of nanoparticles in modern day uses, the costs associated with their synthesis need to be regulated and controlled. Thus, the cost-effectiveness of the production procedure is an important factor that influences nanoparticle synthesis. Although the chemical method of synthesis results in a high yield within a short period of time, this technique is not costeffective. Therefore, synthesis using chemical and physical methods may be limited, whereas biological synthesis of nanoparticles involves less cost and can be performed on a large scale.

3.7. Particle Shape and Size. Particle size plays an important role in determining the properties of nanoparticles. For example, the melting point of nanoparticles has been reported to decrease when the size of the nanoparticles reached the nanometer scale [33]. Nanoparticles with different configurations have similar energy that makes the transformation of their shape easy [34]. The type of energy commonly used during the analysis of the nanoparticles stimulates the change in the shape of the nanoparticle. The dynamic nature and shape of the synthesized nanoparticles greatly affect their chemical properties [35].

3.8. Pore Size. The quality and application of nanoparticles are greatly influenced by the porosity of the synthesized nanoparticles. Immobilization of biomolecules onto nanoparticles has been achieved to increase their use in the drug delivery and biomedical field [36].

3.9. Environment. The surrounding environment plays an important role in determining the nature of the synthesized nanoparticles. In many environments, a single nanoparticle becomes core-shell nanoparticles quickly by absorbing materials or reacting with other materials from the environment by the process of oxidation or corrosion [37]. In a biological system, the synthesized nanoparticles form a coating that makes them thicker and larger-sized [38]. In addition to this, the environment also affects the physical structure and chemistry of the synthesized nanoparticles. There are few examples that showed the effect of the environment on the nature of the synthesized nanoparticles. The crystalline nature of the zinc sulphide nanoparticles changed immediately when its environment was changed from a wet to a dry condition. Similarly, the chemical nature of cerium nitrate nanoparticles varies with the presence of the peroxide in the solution in which they are suspended [30].

3.10. Proximity. When the individual or isolated nanoparticles come in contact or near to the surface of other nanoparticles, alteration in their properties is observed in most of the cases [39]. This changing behavior of the nanoparticles can be utilized in making more tuned nanoparticles. There are many implications of the proximity effect of nanoparticles such as the particle charging, the substrate interactions, and magnetic properties of the nanoparticles.

3.11. Other Factors. Various living systems such as plants are rich in secondary metabolites that act as reducing and stabilizing agents for the synthesis of nanoparticles. However, the composition of these metabolites varies depending on the type of plant, plant part, and the procedure used for its extraction [40]. Similarly, different microorganisms produce distinct intracellular and extracellular enzymes in 
varying quantities that affect nanoparticle synthesis [41]. Furthermore, the choice of method used to purify the synthesized nanoparticles can influence nanoparticle quantity and quality. In some cases, centrifugation is performed to separate the nanoparticles based on gravitational force [21]. In other cases, chromatography techniques are used to separate nanoparticles according to differences between the mobile phase and stationary phase coefficients [42]. Efficient separation of synthesized nanoparticles is achieved by one or more procedures, including the extraction of nanoparticles based on their solubility in two different miscible liquid phases (usually water and organic solvents) followed by electrophoresis or chromatography [43]. Separation of nanoparticles is important for their application in pharmaceutical and biomedical industries [44].

\section{Characterization of the Synthesized Nanoparticles}

The nanoparticles present a range of characterization challenges that affect the detailed and appropriate characterization of nanoparticles. Thus understanding the problems faced during characterization of nanoparticles and selecting a suitable characterization technique are of utmost importance. Specifically, nanoparticle characterization is performed to assess the surface area and porosity, pore size, solubility, particle size distribution, aggregation, hydrated surface analysis, zeta potential, wettability, adsorption potential and shape, size of the interactive surface, crystallinity, fractal dimensions, orientation, and the intercalation and dispersion of nanoparticles and nanotubes in nanocomposite materials [7]. Several techniques can be used to determine nanoparticle parameters, including ultraviolet- (UV-) visible spectroscopy, atomic force microscopy (AFM), transmission electron microscopy (TEM), scanning electron microscopy (SEM), dynamic light scattering (DLS), X-ray photoelectron spectroscopy (XPS), thermogravimetric analysis (TGA), powder $\mathrm{X}$-ray diffraction (XRD), Fourier transform infrared spectroscopy (FT-IR), matrix-assisted laser desorption/ionization time-of-flight mass spectrometry (MALDI-TOF), dual polarization interferometry, nuclear magnetic resonance (NMR), nanoparticle tracking analysis (NTA) for evaluation of Brownian motion, and particle size analysis [45-47]. Some of the important characterization techniques designed to measure specific parameters are described in detail below (Table 1).

4.1. Nanoparticle Formation Analysis. UV-visible spectroscopy is used to confirm the formation of various types of nanoparticles by measuring Plasmon resonance and evaluating the collective oscillations of conduction band electrons in response to electromagnetic waves [7]. This provides information about the size, structure, stability, and aggregation of the nanoparticles [13]. Metal nanoparticles are associated with specific absorbance bands in characteristic spectra when the incident light enters into resonance with the conduction band electrons on the surface of the nanoparticle. For example, silver nanoparticles produce a specific absorbance peak between 400 and $450 \mathrm{~nm}$, while gold nanoparticles have

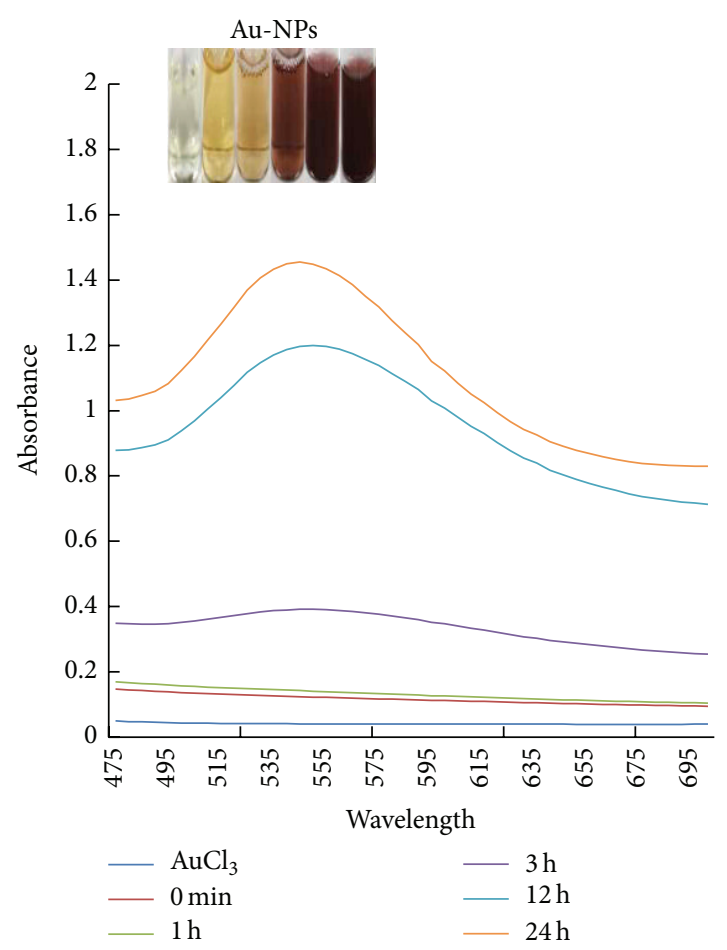

FIGURE 3: The UV-visible spectroscopy of gold nanoparticle.

an absorbance peak between 500 and $550 \mathrm{~nm}$ (Figure 3) due to the excitation mode of the surface plasmons, which vary depending on the size of the nanoparticle [48-50].

4.2. Nanoparticle Extraction Analysis. The extraction of nanoparticles is undertaken by a critical analytical process of Cloud point extraction. Apart from the matrix effects in the environmental samples, the low concentrations of nanoparticles require enrichment procedure prior to its analytical determination that can be obtained by adding a surfactant to the sample at a concentration that exceeds the critical concentration. At higher temperature than the specific cloud point, the surfactants form micelles in which the nonpolar substances are encapsulated since their densities are higher than water; thus they settle down at the bottom of the solution and the nanoparticles are extracted by further centrifugation procedure $[51,52]$.

4.3. Morphology and Particle Size Determination. Morphology and particle size distribution are the most important parameters for characterizing nanoparticles. These factors can be measured by microscopic techniques such as TEM, SEM, and AFM [1]. Most nanoparticle applications or associated factors such as drug targeting and release, tissue targeting, toxicity and biological fate, or in vivo distribution are linked to the size and size distribution of nanoparticles. Various studies have shown that microparticles are less effective for drug delivery than nanoparticles owing to their larger size $[1,53,54]$. Nanoparticles are more effective since they provide large surface areas for drug interaction due to their small size [53]. However, in some cases, aggregation 


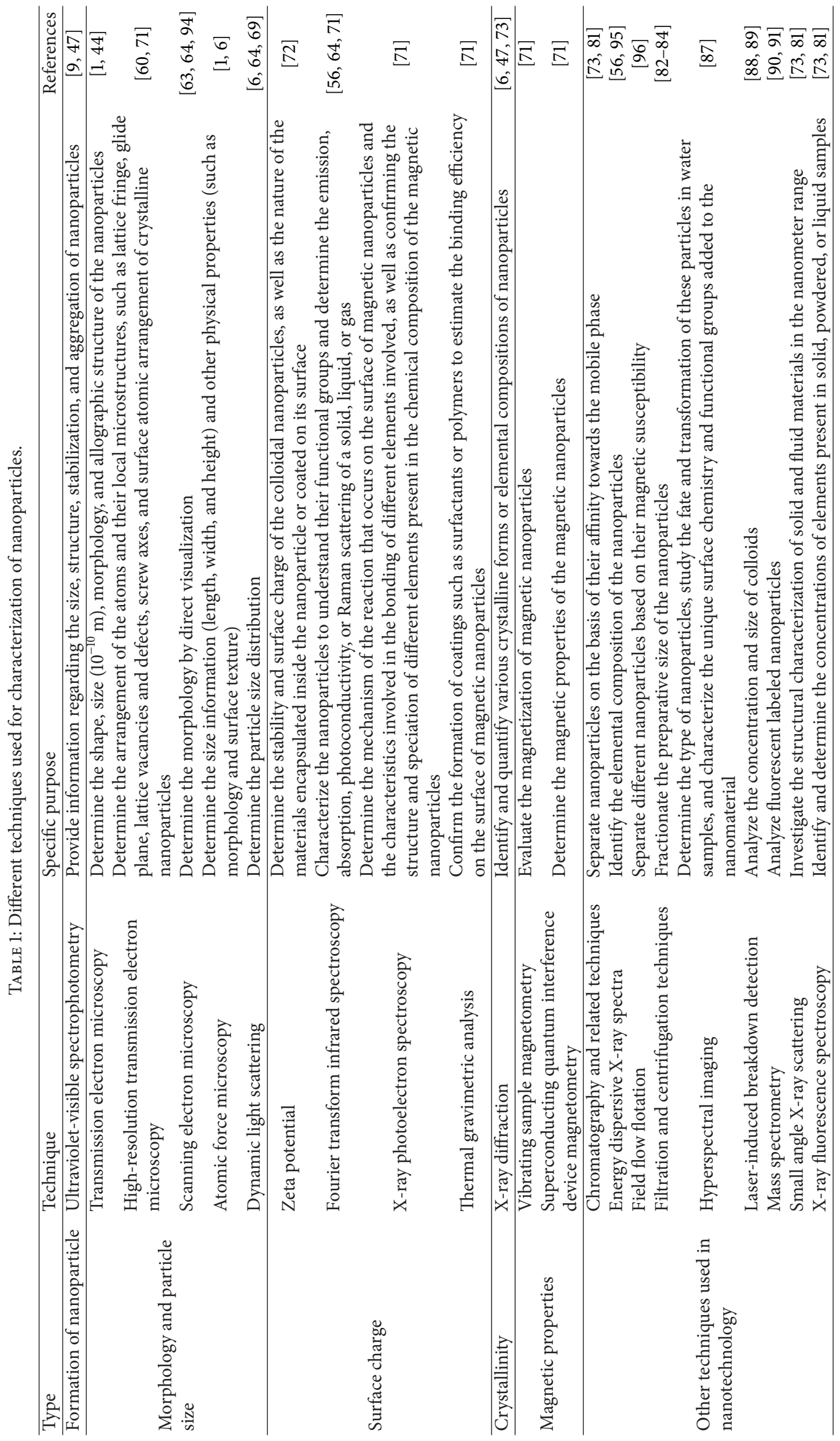




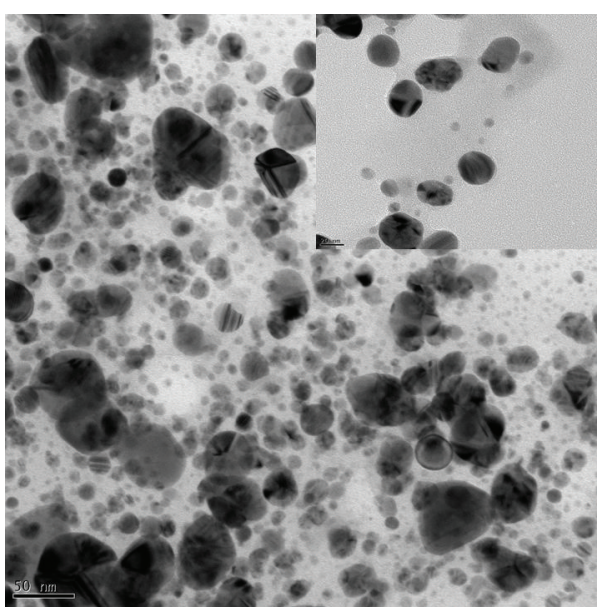

(a)

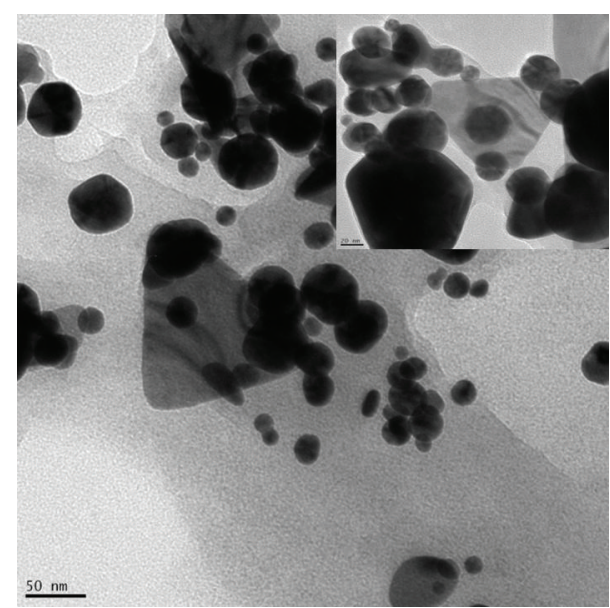

(b)

FIGURE 4: Transmission electron microscopy image of silver (a) and gold (b) nanoparticles.

can occur with small particle size. Therefore, nanoparticles with a relatively large size are thought to promote rapid drug release and more effective polymer degradation $[1,54]$. There are several tools available for determining nanoparticle size, which are presented below.

4.3.1. TEM. TEM is one of the most commonly used methods for determination of the shape, size, and morphology of nanoparticles [6,55]. However, sample preparation for TEM is very complex and time-consuming because the samples must be ultrathin for electron transmittance. Thus, thin films containing the samples are prepared on carbon-coated copper grids by dropping a very small amount of the sample in solution onto the grid and then removing the extra solution with blotting paper. To withstand the vacuum pressure of the microscope and facilitate proper handling, the nanoparticles are fixed using a negative staining solution (phosphotungstic acid) or derivatives (e.g., uranyl acetate), after which they are embedded in plastic or exposed to liquid nitrogen after embedding in vitreous ice [1]. The particles are subsequently allowed to dry under a mercury lamp and then are exposed to a monochromatic beam of electrons that penetrates the sample and is projected onto a viewing screen to generate an image [56-59]. Using TEM, small particles $\left(10^{-10} \mathrm{~m}\right.$ in size, which is near the atomic level) can be viewed and the crystallographic structure of a sample can be imaged at an atomic scale (Figure 4) [1]. Arrangement of the atoms and their local microstructures such as lattice fringe, glide plane, lattice vacancies and defects, screw axes, and the surface atomic arrangement of crystalline nanoparticles can be analyzed using high-resolution transmission electron microscopy (HRTEM) [60].

4.3.2. SEM. SEM is another technique used to characterize the morphology of nanoparticles through direct visualization. This method is based on electron microscopy and offers several advantages for morphological and size analysis; however, it is also associated with several disadvantages, such as the ability to provide only limited information about the size distribution and true population average [1]. The instrument has features that include an electron gun, condenser lenses, and a vacuum system. SEM produces three types of principal images: external X-ray maps, backscattered electron images, and secondary electron images [47]. For SEM analysis, the nanoparticle solution is dried into a powder, mounted on a sample holder, and coated with a conductive metal such as gold, gold/palladium alloy, platinum, osmium, iridium, tungsten, chromium, or graphite using a sputter coater [61]. Next, a beam of high-energy electrons is directed to the sample to generate a variety of signals on the surface of the specimens [62]. The signals received from the sample exposed to electron beams are recorded by a detector that reveals information about the samples, including their texture (external morphology), crystalline structure, and chemical composition and orientation of the materials in the sample $[49,50]$. For a successful analysis, the nanoparticles should be able to withstand vacuum pressure and the adverse effects of the electron beam, which can damage nanopolymers [1]. In most cases, data for a selected area of the surface of the nanomaterial are collected and a two-dimensional image with spatial variations is displayed (Figure 5) [63, 64]. Despite these advantages, this technique is time consuming and costly and often requires complementary information about the size distribution.

4.3.3. AFM. AFM is used to study the morphology of nanoparticles and biomolecules. Unlike SEM and TEM, AFM produces three-dimensional images so that particle volume and height can be evaluated $[65,66]$. This method is capable of ultra-high resolution for particle size measurement and is based on physical scanning of the samples at the submicron level using a probe tip [67]. Using AFM, quantitative information regarding individual nanoparticles and groups of particles such as size (length, width, and height), morphology, and surface texture can be evaluated with the help of softwarebased image processing [6]. AFM can be performed in either 


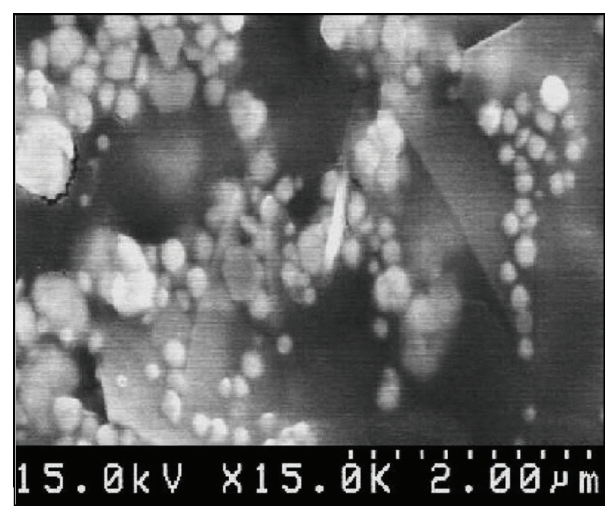

FIGURE 5: Scanning electron microscopy image of gold nanoparticles.

liquid or gas medium. For this method, a small volume of the nanoparticles is spread on a glass cover slip mounted on the AFM stub and dried with nitrogen gas at room temperature. About six to ten images are then taken for a single sample to enable better interpretation of the data. The instrument generates a topographical map of the sample based on the forces between the tip and the surface of the sample, which is scanned in contact mode. The probe hovers over the conducting surface when in noncontact mode, depending on the sample-specific properties [6]. The main advantage of AFM is its ability to image nonconducting samples without any specific treatment and the ability to image delicate biological or polymeric micro- and nanostructures [68].

4.3.4. DLS. DLS, otherwise known as photon-correlation spectroscopy, is one of the fastest and most popular methods for determining particle size distribution. DLS is widely used to measure the size of Brownian nanoparticles in colloidal suspensions $[6,69]$. When a monochromatic beam of light (laser) is directed onto a solution of spherical particles in Brownian motion, a Doppler shift occurs when the light hits the moving particles, thereby changing the wavelength of the incoming beam of light by a value related to particle size. Accordingly, DLS enables computation of size distribution and nanoparticle motion in the medium can be computed by measuring the diffusion coefficient of the particle [57].

4.3.5. Nanoparticle Tracking Analysis. The nanoparticle tracking analysis is an improved system that is used to categorize different types of nanoparticles on the basis of their size ranging between 30 and $1000 \mathrm{~nm}$ with the lower detection limit depending on its refractive index. With the help of this technique, the liquid nanoparticle suspension can be visualized directly and it has application in drug delivery encapsulated nanoparticles for controlled release or precise delivery of the drug to the specific targeted areas [70].

4.4. Surface Charge Analysis. Another important parameter for characterizing nanoparticles is surface charge. The nature and intensity of the surface charge are very important since these factors determine the interaction of the nanoparticle

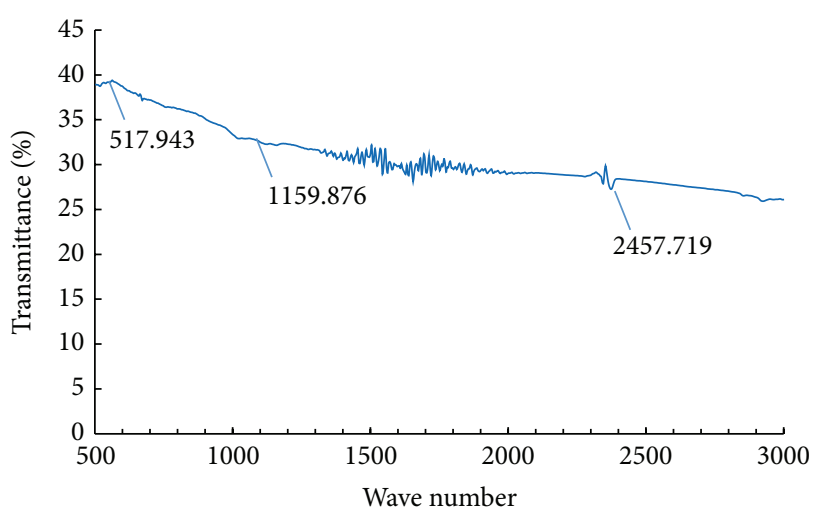

FIGURE 6: FT-IR spectra of silver nanoparticles.

with the biological environment and the electrostatic interactions with the bioactive compounds from plants, algae, fungi, and bacteria.

4.5. XPS. XPS is used to study the mechanism of reaction that occurs on the surface of magnetic nanoparticles, assess the bonding characteristics of the different elements involved, and confirm the structure and different elements present in the magnetic nanoparticles [71].

4.6. FT-IR Spectroscopy. FT-IR spectroscopy is conducted to identify the functional groups present on nanoparticles. Using FT-IR analysis, the infrared emission spectrum, absorption, photoconductivity, or Raman scattering of a solid, liquid, or gas can be evaluated. The spectrum represents a fingerprint of the nanoparticles consisting of absorption peaks that correspond to the frequencies of vibrations between the bonds of atoms in the nanoparticle (Figure 6). Since each type of nanoparticle contains a unique combination of atoms, we can identify functional groups present inside the nanoparticles based on the FT-IR spectra [71]. This can help facilitate nanoparticle synthesis using green technology. The number of functional groups present in the nanomaterial can be determined by the size of the peaks of the spectrum $[6,71]$. The transmission spectra for the nanoparticles are obtained by the formation of thin, transparent potassium bromide $(\mathrm{KBr})$ pellets containing the compound of interest. The $\mathrm{KBr}$ mixtures are placed in a vacuum line overnight prior to pellet formation, and the pellets are again placed in the vacuum line before use. The transmission spectra are obtained after purging in dry air and background corrected relative to a reference blank sample $(\mathrm{KBr})$ [47]. With the application of modern software tools, quantitative analysis of the nanoparticles can be completed within a few seconds $[56,64]$.

4.7. Zetasizer Nanomachine. The stability and surface charge of colloidal nanoparticles are evaluated indirectly by performing zeta potential analysis using a Zetasizer nanomachine. Zeta potential analysis corresponds to the potential difference between the outer Helmholtz plane and the surface 
of shear. Measurement of the zeta potential predicts the storage stability of the colloidal dispersion. Either high positive or negative zeta potential values should be achieved to ensure stability and avoid particle aggregation. Additionally, the extent of surface hydrophobicity can be predicted. The nature of the materials encapsulated inside the nanoparticle or coated on the particle surface is also analyzed based on zeta potential [72].

4.8. TGA. TGA is used to confirm the composition of coatings such as surfactants or polymers to estimate the binding efficiency on the surface of magnetic nanoparticles [71].

4.9. Crystallinity Analysis. XRD is used to assess the crystallinity of synthesized nanoparticles [6]. This technique is employed to identify and quantitatively examine various crystalline forms or the elemental composition of natural and manufactured materials or nanoparticles $[6,73]$. To accomplish this, the structure and lattice parameters of the diffracted powder specimen are analyzed by measuring the angle of diffraction, when X-ray beam are made to incident on them. Particle size is also determined based on the width of the X-ray peaks using the Scherrer formula [47].

4.10. Surface Hydrophobicity Assessment. Surface hydrophobicity of the nanoparticles can be measured using several analytical techniques, including biphasic partitioning, probe adsorption, hydrophobic interaction chromatography, and contact angle measurements. X-ray photon correlation spectroscopy has recently been used to identify specific chemical groups on the surface of nanoparticles [74].

4.11. Analysis of Nanoparticle Magnetic Properties. Many techniques are available to investigate the properties of magnetic nanoparticles including vibrating sample magnetometry (VSM) and superconducting quantum interference device (SQUID) magnetometry [71]. However, neither of these techniques is element-specific, and they can only measure general magnetization. SQUID magnetometry is routinely used to assess the properties of magnetic nanoparticles. To accomplish this, nanoparticles are cooled with or without an applied magnetic field and then warmed in the presence of a magnetic field [71]. Magnetization is monitored as a function of temperature.

VSM is conducted to evaluate the magnetization of magnetic nanoparticles as a function of an applied external magnetic field $(H)$, generally between -3 and 3 Tesla. Based on the VSM curve obtained at low and room temperature, the magnetic behavior of the nanoparticles can be observed. VSM is a good technique for estimating the effects of a shell on saturation magnetization [71].

4.12. Other Techniques. Apart from the above methods used for specific characterization of nanoparticles, there are a number of other techniques that can be employed to characterize, fractionate, and separate nanoparticles for specific purposes.
4.12.1. Energy Dispersive X-Ray Spectra (EDX). EDX analysis is used to characterize nanoparticles synthesized with green technology. To accomplish this, X-rays are emitted from nanoparticles after being bombarded by an electron beam. The elemental composition of the nanoparticles can then be determined. An EDS detector that can be attached to a scanning electron microscope can detect the number of $\mathrm{X}$-rays which are emitted to balance the energy difference between two electrons. The energy of the emitted X-ray is a characteristic feature of the element, and the element is therefore analyzed qualitatively and quantitatively $[56,75]$.

4.12.2. Chromatography and Related Techniques. There are different chromatography-based protocols used to separate nanoparticles in solution. These procedures are sensitive, rapid, and nondestructive [53]. Size exclusion chromatography (SEC) is the best known technique for separating nanoparticles (quantum dots, single-walled carbon nanotubes, and polystyrene nanoparticles) based on size [7678]. Hydrodynamic chromatography (HDC) separates particles according to their hydrodynamic radius $[73,79]$. The available HDC columns have a size separation range of 5 to $1200 \mathrm{~nm}$ depending on the column length. This relatively wide separation range allows nanoparticles of various sizes to be measured in different media and is particularly helpful for monitoring the formation of aggregates [73]. Field-flow fractionation is another highly promising method for separating elemental nanoparticles in complex natural samples according to size [80]. This method is similar to chromatographic techniques, but nanoparticle separation is solely conducted in an open channel without relying on a stationary phase. The particles are separated based on how they are affected by an applied field $[73,81]$.

4.12.3. Filtration and Centrifugation Techniques. Centrifugation and filtration procedures are two important techniques employed for the preparative size fractionation of nanoparticles. These methods are cost-effective and highly efficient. Preparative ultracentrifugation is used to pellet fine particulate fractions and the separation and harvesting of aquatic colloids or nanoparticles on TEM and AFM substrates [82-84]. Traditional membrane filtration is used to separate nanoparticles and ions with pore sizes of 0.5 or $1 \mathrm{~nm}$ $[85,86]$.

4.12.4. Field Flow Flotation. In the case of magnetic nanoparticles, it is difficult to synthesize monodispersed particulate materials. Therefore, polydispersed magnetic nanoparticles are characterized using magnetic field flow fractionation (MFFF), in which species are separated based on magnetic susceptibility. This technique is appropriate for materials with sizes in the nanometer to micrometer ranges [71].

4.12.5. Hyperspectral Imaging. Hyperspectral imaging is conducted to determine the type of nanoparticles synthesized in environmental systems and facilitate investigations of the fate and transformation of these particles in water. Additionally, unique surface chemistry and functional groups added to 
the nanomaterials can be characterized. Data describing sample information are presented as spatial distributions and spectral characteristics unique to each nanoparticle type at the sensitivity of a single nanoparticle $($ size $<10 \mathrm{~nm})$ [87].

4.12.6. Laser-Induced Breakdown Detection (LIBD). LIBD is a laser-based technique with extremely low detection limits capable of analyzing the concentration and size of colloids depending on the measured breakdown probability (BP). Laser-induced breakdown detection is a highly promising tool for nanoparticle characterization [73, 88, 89]. Other laser-based methods include mass spectrometry (MS), laserinduced fluorescence (LIF), and Raman spectroscopy [73]. MS-based procedures such as MALDI, LIF, or ion trap mass spectrometry are used to analyze fluorescent-labeled nanoparticles [90, 91].

4.12.7. Small Angle X-Ray Scattering (SAXS). SAXS is an analytical X-ray-based technique employed to investigate the structural characteristics of solid and fluid materials in the nanometer range [81]. This nondestructive procedure is able to identify elements and determine the concentrations present in solid, powdered, or liquid samples [81].

4.12.8. Inductively Coupled Plasma Mass Spectrometry (ICP$M S$ ). ICP-MS is used for determination of ultra-stress metals in a wide range of samples. It is also used to determine the toxicity of nanoparticles for their potential use in biomedical application [92]. Different types of nanoparticles like gold, nickel, and single walled carbon nanotubes are quantified by the ICP-MS technique [93].

\section{Conclusions}

The use of nanoparticles in the medicinal, food, pharmaceutical, and agricultural industries has garnered a great deal of interest, with a focus on development of more convenient methods using green biotechnology tools for production of eco-friendly, nontoxic, and environmentally benign nanoparticles. There are many factors that influence the quality and quantity of synthesized nanoparticles for their potential use in various applications. Suitable characterization techniques that can characterize the synthesized nanoparticles efficiently and thus increase their use in environmental, electronic, and biomedical applications and drug delivery applications are needed. In this review, different factors influencing the synthesis of nanoparticles and various techniques that are used to characterize them were described in detail. Current and future investigations of green nanotechnology will provide a more complete knowledge base regarding various factors that influence green synthesis of nanoparticles and the most sophisticated technology that can be used for characterization of the synthesized nanoparticles for its more efficient future applications in biomedical and pharmaceutical industries.

\section{Conflict of Interests}

The authors declare that there is no conflict of interests regarding the publication of this paper.

\section{Acknowledgment}

This research was supported by a Grant (NRF2014R1A1A2A16053256) from the Basic Science Research Program of the National Research Foundation of Republic of Korea.

\section{References}

[1] S. L. Pal, U. Jana, P. K. Manna, G. P. Mohanta, and R. Manavalan, "Nanoparticle: an overview of preparation and characterization," Journal of Applied Pharmaceutical Science, vol. 1, no. 6, pp. 228-234, 2011.

[2] A. Cauerhff and G. R. Castro, "Bionanoparticles, a green nanochemistry approach," Electronic Journal of Biotechnology, vol. 16, no. 3, 2013.

[3] M. Faraday, "Experimental relations of gold (and other metals) to light," Philosophical Transactions of the Royal Society B, vol. 147, pp. 145-181, 1987.

[4] M. Singh, S. Manikandan, and A. K. Kumaraguru, "Nanoparticles: a new technology with wide applications," Research Journal of Nanoscience and Nanotechnology, vol. 1, no. 1, pp. 1-11, 2011.

[5] R. Joerger, T. Klaus, and C. G. Granqvist, "Biologically produced silver carbon composite materials for optically functional thinfilm coatings," Advanced Materials, vol. 12, no. 6, pp. 407-409, 2000.

[6] R. P. S. Chauhan, C. Gupta, and D. Prakash, "Methodological advancements in green nanotechnology and their applications in biological synthesis of herbal nanoparticles," International Journal of Bioassays, vol. 1, no. 7, pp. 6-10, 2012.

[7] A. G. Ingale and A. N. Chaudhari, "Biogenic synthesis of nanoparticles and potential applications: an eco-friendly approach," Journal of Nanomedicine and Nanotechnology, vol. 4, no. 2, 2013.

[8] P. Mohanpuria, N. K. Rana, and S. K. Yadav, "Biosynthesis of nanoparticles: technological concepts and future applications," Journal of Nanoparticle Research, vol. 10, no. 3, pp. 507-517, 2008.

[9] D. K. Tiwari, J. Behari, and P. Sen, “Time and dose-dependent antimicrobial potential of Ag nanoparticles synthesized by topdown approach," Current Science, vol. 95, no. 5, pp. 647-655, 2008.

[10] N. A. Luechinger, R. N. Grass, E. K. Athanassiou, and W. J. Stark, "Bottom-up fabrication of metal/metal nanocomposites from nanoparticles of immiscible metals," Chemistry of Materials, vol. 22, no. 1, pp. 155-160, 2010.

[11] U. K. Parashar, P. S. Saxena, and A. Srivastava, "Bioinspired synthesis of silver nanoparticles," Digest Journal of Nanomaterials and Biostructures, vol. 4, no. 1, pp. 159-166, 2009.

[12] V. Parashar, R. Parashar, B. Sharma, and A. C. Pandey, "Partenium leaf extract mediated synthesis of silver nanoparticles: a novel approach towards weed utilization," Digest Journal of Nanomaterials and Biostructures, vol. 4, no. 1, pp. 45-50, 2009.

[13] M.-C. Daniel and D. Astruc, "Gold nanoparticles: assembly, supramolecular chemistry, quantum-size-related properties, and applications toward biology, catalysis, and nanotechnology," Chemical Reviews, vol. 104, no. 1, pp. 293-346, 2004. 
[14] X. Li, H. Xu, Z.-S. Chen, and G. Chen, "Biosynthesis of nanoparticles by microorganisms and their applications," Journal of Nanomaterials, vol. 2011, Article ID 270974, 16 pages, 2011.

[15] P. T. Anastas and J. C. Warner, Green Chemistry: Theory and Practice, Oxford University Press, New York, NY, USA, 1998.

[16] O. V. Kharissova, H. V. R. Dias, B. I. Kharisov, B. O. Pérez, and V. M. J. Pérez, "The greener synthesis of nanoparticles," Trends in Biotechnology, vol. 31, no. 4, pp. 240-248, 2013.

[17] K. B. Narayanan and N. Sakthivel, "Green synthesis of biogenic metal nanoparticles by terrestrial and aquatic phototrophic and heterotrophic eukaryotes and biocompatible agents," Advances in Colloid and Interface Science, vol. 169, no. 2, pp. 59-79, 2011.

[18] P. M. Ajayan, "Nanotechnology: how does a nanofibre grow?" Nature, vol. 427, no. 6973, pp. 402-403, 2004.

[19] G. A. Somorjai and J. Y. Park, "Colloid science of metal nanoparticle catalysts in 2D and 3D structures. Challenges of nucleation, growth, composition, particle shape, size control and their influence on activity and selectivity," Topics in Catalysis, vol. 49, no. 3-4, pp. 126-135, 2008.

[20] T. J. Pennycook, J. R. McBride, S. J. Rosenthal, S. J. Pennycook, and S. T. Pantelides, "Dynamic fluctuations in ultrasmall nanocrystals induce white light emission," Nano Letters, vol. 12, no. 6, pp. 3038-3042, 2012.

[21] S. Baker, D. Rakshith, K. S. Kavitha et al., "Plants: emerging as nanofactories towards facile route in synthesis of nanoparticles," BioImpacts, vol. 3, no. 3, pp. 111-117, 2013.

[22] V. Vadlapudi and D. S. V. G. K. Kaladhar, "Review: green synthesis of silver and gold nanoparticles," Middle-East Journal of Scientific Research, vol. 19, no. 6, pp. 834-842, 2014.

[23] J. L. Gardea-Torresdey, K. J. Tiemann, G. Gamez, K. Dokken, and N. E. Pingitore, "Recovery of gold (III) by alfalfa biomass and binding characterization using X-ray microfluorescence," Advances in Environmental Research, vol. 3, no. 1, pp. 83-93, 1999.

[24] V. Armendariz, I. Herrera, J. R. Peralta-Videa et al., "Size controlled gold nanoparticle formation by Avena sativa biomass: use of plants in nanobiotechnology," Journal of Nanoparticle Research, vol. 6, no. 4, pp. 377-382, 2004.

[25] N. Soni and S. Prakash, "Factors affecting the geometry of silver nanoparticles synthesis in Chrysosporium tropicum and Fusarium oxusporum," American Journal of Nanotechnology, vol. 2, no. 1, pp. 112-121, 2011.

[26] A. Rai, A. Singh, A. Ahmad, and M. Sastry, "Role of halide ions and temperature on the morphology of biologically synthesized gold nanotriangles," Langmuir, vol. 22, no. 2, pp. 736-741, 2006.

[27] B. D. P. Abhilash, "Synthesis of zinc-based nanomaterials: a biological perspective," IET Nanobiotechnology, vol. 6, no. 4, pp. 144-148, 2012.

[28] Q. H. Tran, V. Q. Nguyen, and A. T. Le, "Silver nanoparticles: synthesis, properties, toxicology, applications and perspectives," Advances in Natural Sciences: Nanoscience and Nanotechnology, vol. 4, Article ID 033001, 2013.

[29] M. Darroudi, M. B. Ahmad, R. Zamiri, A. K. Zak, A. H. Abdullah, and N. A. Ibrahim, "Time-dependent effect in green synthesis of silver nanoparticles," International Journal of Nanomedicine, vol. 6, no. 1, pp. 677-681, 2011.

[30] S. V. N. T. Kuchibhatla, A. S. Karakoti, D. R. Baer et al., "Influence of aging and environment on nanoparticle chemistry: implication to confinement effects in nanoceria," Journal of Physical Chemistry C, vol. 116, no. 26, pp. 14108-14114, 2012.
[31] I. A. Mudunkotuwa, J. M. Pettibone, and V. H. Grassian, "Environmental implications of nanoparticle aging in the processing and fate of copper-based nanomaterials," Environmental Science and Technology, vol. 46, no. 13, pp. 7001-7010, 2012.

[32] D. R. Baer, "Surface characterization of nanoparticles: critical needs and significant challenges," Journal of Surface Analysis, vol. 17, no. 3, pp. 163-169, 2011.

[33] B. Akbari, M. P. Tavandashti, and M. Zandrahimi, "Particle size characterization of nanoparticles-a practicalapproach," Iranian Journal of Materials Science and Engineering, vol. 8, no. 2, pp. 48-56, 2011.

[34] M. J. Yacamán, J. A. Ascencio, H. B. Liu, and J. GardeaTorresdey, "Structure shape and stability of nanometric sized particles," Journal of Vacuum Science and Technology B, vol. 19, no. 4, pp. 1091-1103, 2001.

[35] D. R. Baer, M. H. Engelhard, G. E. Johnson et al., "Surface characterization of nanomaterials and nanoparticles: important needs and challenging opportunities," Journal of Vacuum Science \& Technology A, vol. 31, no. 5, Article ID 050820, 2013.

[36] E. Ruckenstein and X. Z. Kong, "Control of pore generation and pore size in nanoparticles of poly(styrene-methyl methacrylateacrylic acid)," Journal of Applied Polymer Science, vol. 72, no. 3, pp. 419-426, 1999.

[37] V. Sarathy, P. G. Tratnyek, J. T. Nurmi et al., "Aging of iron nanoparticles in aqueous solution: effects on structure and reactivity," The Journal of Physical Chemistry C, vol. 112, no. 7, pp. 2286-2293, 2008.

[38] I. Lynch, T. Cedervall, M. Lundqvist, C. Cabaleiro-Lago, S. Linse, and K. A. Dawson, "The nanoparticle-protein complex as a biological entity; a complex fluids and surface science challenge for the 21st century," Advances in Colloid and Interface Science, vol. 134-135, pp. 167-174, 2007.

[39] D. R. Baer, J. E. Amonette, M. H. Engelhard et al., "Characterization challenges for nanomaterials," Surface and Interface Analysis, vol. 40, no. 3-4, pp. 529-537, 2008.

[40] Y. Park, Y. N. Hong, A. Weyers, Y. S. Kim, and R. J. Linhardt, "Polysaccharides and phytochemicals: a natural reservoir for the green synthesis of gold and silver nanoparticles," IET Nanobiotechnology, vol. 5, no. 3, pp. 69-78, 2011.

[41] R. G. Haverkamp, A. T. Marshall, and D. Van Agterveld, "Pick your carats: nanoparticles of gold-silver-copper alloy produced in vivo," Journal of Nanoparticle Research, vol. 9, no. 4, pp. 697700, 2007.

[42] V. L. Jimenez, M. C. Leopold, C. Mazzitelli, J. W. Jorgenson, and R. W. Murray, "HPLC of monolayer-protected gold nanoclusters," Analytical Chemistry, vol. 75, no. 2, pp. 199-206, 2003.

[43] M. Hanauer, S. Pierrat, I. Zins, A. Lotz, and C. Sönnichsen, "Separation of nanoparticles by gel electrophoresis according to size and shape," Nano Letters, vol. 7, no. 9, pp. 2881-2885, 2007.

[44] B. Kowalczyk, I. Lagzi, and B. A. Grzybowski, "Nanoseparations: strategies for size and/or shape-selective purification of nanoparticles," Current Opinion in Colloid and Interface Science, vol. 16, no. 2, pp. 135-148, 2011.

[45] G. B. Khomutov and S. P. Gubin, "Interfacial synthesis of noble metal nanoparticles," Materials Science and Engineering C, vol. 22, no. 2, pp. 141-146, 2002.

[46] Y. Choi, N.-H. Ho, and C.-H. Tung, "Sensing phosphatase activity by using gold nanoparticles," Angewandte Chemie, vol. 46, no. 5, pp. 707-709, 2007.

[47] V. Gupta, A. R. Gupta, and V. Kant, "Synthesis, characterization and biomedical application of nanoparticles," Science International, vol. 1, no. 5, pp. 167-174, 2013. 
[48] P. V. Asharani, Y. L. Wu, Z. Gong, and S. Valiyaveettil, “Toxicity of silver nanoparticles in zebrafish models," Nanotechnology, vol. 19, no. 25, Article ID 255102, 2008.

[49] M. Linga Rao and N. Savithramma, "Biological synthesis of silver nanoparticles using Svensonia Hyderabadensis leaf extract and evaluation of their antimicrobial efficacy," Journal of Pharmaceutical Sciences and Research, vol. 3, no. 3, pp. 1117-1121, 2011.

[50] N. N. Devi, P. D. Shankar, W. Femina, and T. Paramasivam, "Antimicrobial efficacy of green synthesized silver nanoparticles from the medicinal plant Plectranthus amboinicus," International Journal of Pharmaceutical Sciences Review and Research, vol. 12, no. 1, pp. 164-168, 2012.

[51] J.-B. Chao, J.-F. Liu, S.-J. Yu et al., "Speciation analysis of silver nanoparticles and silver ions in antibacterial products and environmental waters via cloud point extraction-based separation," Analytical Chemistry, vol. 83, no. 17, pp. 6875-6882, 2011.

[52] G. Hartmann, C. Hutterer, and M. Schuster, "Ultra-trace determination of silver nanoparticles in water samples using cloud point extraction and ETAAS," Journal of Analytical Atomic Spectrometry, vol. 28, no. 4, pp. 567-572, 2013.

[53] J. Kreuter, "Peroral administration of nanoparticles," Advanced Drug Delivery Reviews, vol. 7, no. 1, pp. 71-86, 1991.

[54] J. Panyam, M. M. Dali, S. K. Sahoo et al., "Polymer degradation and in vitro release of a model protein from poly(D,L-lactideco-glycolide) nano- and microparticles," Journal of Controlled Release, vol. 92, no. 1-2, pp. 173-187, 2003.

[55] M. Zargar, A. A. Hamid, F. A. Bakar et al., "Green synthesis and antibacterial effect of silver nanoparticles using Vitex negundo L," Molecules, vol. 16, no. 8, pp. 6667-6676, 2011.

[56] K. S. Prasad, D. Pathak, A. Patel et al., "Biogenic synthesis of silver nanoparticles using Nicotiana tobaccum leaf extract and study of their antibacterial effect," African Journal of Biotechnology, vol. 10, no. 41, pp. 8122-8130, 2011.

[57] A. Saxena, R. M. Tripathi, and R. P. Singh, "Biological synthesis of silver nanoparticles by using onion (Allium cepa) extract and their antibacterial activity," Digest Journal of Nanomaterials and Biostructures, vol. 5, no. 2, pp. 427-432, 2010.

[58] K. Mallikarjuna, G. Narasimha, G. R. Dillip et al., "Green synthesis of silver nanoparticles using Ocimum leaf extract and their characterization," Digest Journal of Nanomaterials and Biostructures, vol. 6, no. 1, pp. 181-186, 2011.

[59] K. Vahabi, G. A. Mansoori, and S. Karimi, "Biosynthesis of silver nanoparticles by fungus Trichoderma reesei (a route for largescale production of (AgNPs)," Insciences Journal, vol. 1, no. 1, pp. 65-79, 2011.

[60] S. Brice-Profeta, M.-A. Arrio, E. Tronc et al., "Magnetic order in $\gamma$ - $\mathrm{Fe}_{2} \mathrm{O}_{3}$ nanoparticles: a XMCD study," Journal of Magnetism and Magnetic Materials, vol. 288, pp. 354-365, 2005.

[61] E. Suzuki, "High-resolution scanning electron microscopy of immunogold-labelled cells by the use of thin plasma coating of osmium," Journal of Microscopy, vol. 208, no. 3, pp. 153-157, 2002.

[62] K. Jores, W. Mehnert, M. Drechsler, H. Bunjes, C. Johann, and K. Mäder, "Investigations on the structure of solid lipid nanoparticles (SLN) and oil-loaded solid lipid nanoparticles by photon correlation spectroscopy, field-flow fractionation and transmission electron microscopy," Journal of Controlled Release, vol. 95, no. 2, pp. 217-227, 2004.

[63] S. Prashanth, I. Menaka, R. Muthezhilan, and N. K. Sharma, "Synthesis of plant-mediated silver nanoparticles using medicinal plant extract and evaluation of its antimicrobial activities,"
International Journal of Engineering Science and Technology, vol. 3, no. 8, pp. 6235-6250, 2011.

[64] M. M. Priya, B. K. Selvi, and J. A. J. Paul, "Green synthesis of silver nanoparticles from the leaf extracts of euphorbia hirta and nerium indicum," Digest Journal of Nanomaterials and Biostructures, vol. 6, no. 2, pp. 869-877, 2011.

[65] M. R. Mucalo, C. R. Bullen, M. Manley-Harris, and T. M. McIntire, "Arabinogalactan from the Western larch tree: a new, purified and highly water-soluble polysaccharide-based protecting agent for maintaining precious metal nanoparticles in colloidal suspension," Journal of Materials Science, vol. 37, no. 3, pp. 493-504, 2002.

[66] J. Vesenka, S. Manne, R. Giberson, T. Marsh, and E. Henderson, "Colloidal gold particles as an incompressible atomic force microscope imaging standard for assessing the compressibility of biomolecules," Biophysical Journal, vol. 65, no. 3, pp. 992-997, 1993.

[67] A. Zur Mühlen, E. Zur Mühlen, H. Niehus, and W. Mehnert, "Atomic force microscopy studies of solid lipid nanoparticles," Pharmaceutical Research, vol. 13, no. 9, pp. 1411-1416, 1996.

[68] H. G. Shi, L. Farber, J. N. Michaels et al., "Characterization of crystalline drug nanoparticles using atomic force microscopy and complementary techniques," Pharmaceutical Research, vol. 20, no. 3, pp. 479-484, 2003.

[69] N. De Jaeger, H. Demeyere, R. Finsy et al., "Particle sizing by photon correlation spectroscopy. Part I. Monodisperse latices. Influence of scattering angle and concentration of dispersed material," Particle \& Particle Systems Characterization, vol. 8, no. 1-4, pp. 179-186, 1991.

[70] V. Filipe, A. Hawe, and W. Jiskoot, "Critical evaluation of nanoparticle tracking analysis (NTA) by NanoSight for the measurement of nanoparticles and protein aggregates," Pharmaceutical Research, vol. 27, no. 5, pp. 796-810, 2010.

[71] M. Faraji, Y. Yamini, and M. Rezaee, "Magnetic nanoparticles: synthesis, stabilization, functionalization, characterization, and applications," Journal of the Iranian Chemical Society, vol. 7, no. 1, pp. 1-37, 2010.

[72] H. Otsuka, Y. Nagasaki, and K. Kataoka, "PEGylated nanoparticles for biological and pharmaceutical applications," Advanced Drug Delivery Reviews, vol. 55, no. 3, pp. 403-419, 2003.

[73] K. Tiede, A. B. A. Boxall, S. P. Tear, J. Lewis, H. David, and M. Hassellov, "Detection and characterization of engineered nanoparticles in food and the environment," Food Additives and Contaminants A, vol. 25, no. 7, pp. 795-821, 2008.

[74] P. D. Scholes, A. G. A. Coombes, L. Illum et al., "Detection and determination of surface levels of poloxamer and PVA surfactant on biodegradable nanospheres using SSIMS and XPS," Journal of Controlled Release, vol. 59, no. 3, pp. 261-278, 1999.

[75] D. Mubarak Ali, M. Sasikala, M. Gunasekaran, and N. Thajuddin, "Biosynthesis and characterization of silver nanoparticles using marine cyanobacterium, Oscillatoria willei ntdm01," Digest Journal of Nanomaterials and Biostructures, vol. 6, no. 2, pp. 385-390, 2011.

[76] K. M. Krueger, A. M. Al-Somali, J. C. Falkner, and V. L. Colvin, "Characterization of nanocrystalline CdSe by size exclusion chromatography," Analytical Chemistry, vol. 77, no. 11, pp. 35113515, 2005.

[77] K. J. Ziegler, D. J. Schmidt, U. Rauwald et al., "Lengthdependent extraction of single-walled carbon nanotubes," Nano Letters, vol. 5, no. 12, pp. 2355-2359, 2005. 
[78] X. Huang, R. S. Mclean, and M. Zheng, "High-resolution length sorting and purification of DNA-wrapped carbon nanotubes by size-exclusion chromatography," Analytical Chemistry, vol. 77, no. 19, pp. 6225-6228, 2005.

[79] G. R. McGowan and M. A. Langhorst, "Development and application of an integrated, high-speed, computerized hydrodynamic chromatograph," Journal of Colloid And Interface Science, vol. 89, no. 1, pp. 94-106, 1982.

[80] M. Schimpf, K. Caldwell, and J. C. Giddings, Field-Flow Fractionation Handbook, John Wiley \& Sons, New York, NY, USA, 2000.

[81] A. López-Serrano, R. M. Olivas, J. S. Landaluze, and C. Cámara, "Nanoparticles: a global vision. Characterization, separation, and quantification methods. Potential environmental and health impact," Analytical Methods, vol. 6, no. 1, pp. 38-56, 2014.

[82] A. Bootz, V. Vogel, D. Schubert, and J. Kreuter, "Comparison of scanning electron microscopy, dynamic light scattering and analytical ultracentrifugation for the sizing of poly(butyl cyanoacrylate) nanoparticles," European Journal of Pharmaceutics and Biopharmaceutics, vol. 57, no. 2, pp. 369-375, 2004.

[83] D. Mavrocordatos, D. Perret, and G. G. Leppard, "Strategies and advances in the characterization of environmental colloids by electron microscopy," in Environmental Colloids and Particles: Behaviour, Structure and Characterization, K. J. Wilkinson and J. R. Lead, Eds., pp. 345-404, John Wiley \& Sons, Chichester, UK, 2007.

[84] E. Balnois, G. Papastavrou, and K. J. Wilkinson, "Force microscopy and force measurements of environmental colloids," in Environmental Colloids and Particles: Behaviour, Structure and Characterization, K. J. Wilkinson and J. R. Lead, Eds., pp. 405-468, John Wiley \& Sons, Chichester, UK, 2007.

[85] J. R. Lead and K. J. Wilkinson, "Aquatic colloids and nanoparticles: current knowledge and future trends," Environmental Chemistry, vol. 3, no. 3, pp. 159-171, 2006.

[86] R. Liu and J. R. Lead, "Partial validation of cross flow ultrafiltration by atomic force microscopy," Analytical Chemistry, vol. 78, no. 23, pp. 8105-8112, 2006.

[87] A. R. Badireddy, M. R. Wiesner, and J. Liu, "Detection, characterization, and abundance of engineered nanoparticles in complex waters by hyperspectral imagery with enhanced darkfield microscopy," Environmental Science \& Technology, vol. 46, no. 18, pp. 10081-10088, 2012.

[88] T. Bundschuh, R. Knopp, and J. I. Kim, "Laser-induced breakdown detection (LIBD) of aquatic colloids with different laser systems," Colloids and Surfaces A: Physicochemical and Engineering Aspects, vol. 177, no. 1, pp. 47-55, 2001.

[89] T. Bundschuh, J.-I. Yun, and R. Knopp, "Determination of size, concentration and elemental composition of colloids with laser-induced breakdown detection/spectroscopy (LIBD/S)," Fresenius' Journal of Analytical Chemistry, vol. 371, no. 8, pp. 1063-1069, 2001.

[90] W.-P. Peng, Y. Cai, Y. T. Lee, and H.-C. Chang, "Laser-induced fluorescence/ion trap as a detector for mass spectrometric analysis of nanoparticles," International Journal of Mass Spectrometry, vol. 229, no. 1-2, pp. 67-76, 2003.

[91] Y. Cai, W.-P. Peng, and H.-C. Chang, "Ion trap mass spectrometry of fluorescently labeled nanoparticles," Analytical Chemistry, vol. 75, no. 8, pp. 1805-1811, 2003.

[92] R. S. H. Yang, L. W. Chang, J.-P. Wu et al., "Persistent tissue kinetics and redistribution of nanoparticles, quantum Dot
705, in Mice: ICP-MS quantitative assessment," Environmental Health Perspectives, vol. 115, no. 9, pp. 1339-1343, 2007.

[93] C. Ispas, D. Andreescu, A. Patel, D. V. Goia, S. Andreescu, and K. N. Wallace, "Toxicity and developmental defects of different sizes and shape nickel nanoparticles in zebrafish," Environmental Science and Technology, vol. 43, no. 16, pp. 63496356, 2009.

[94] J. Molpeceres, M. R. Aberturas, and M. Guzman, "Biodegradable nanoparticles as a delivery system for cyclosporine: preparation and characterization," Journal of Microencapsulation, vol. 17, no. 5, pp. 599-614, 2000.

[95] W. R. Herguth and G. Nadeau, Applications of Scanning Electron Microscopy and Energy Dispersive Spectroscopy (SEM/EDS) to Practical Tribology Problems, SGS Herguth Laboratories, Vallejo, Calif, USA, 2004.

[96] T. M. Vickrey and J. A. Garcia-Ramirez, "Magnetic fieldflow fractionation: theoretical basis," Separation Science and Technology, vol. 15, no. 6, pp. 1297-1304, 1980. 

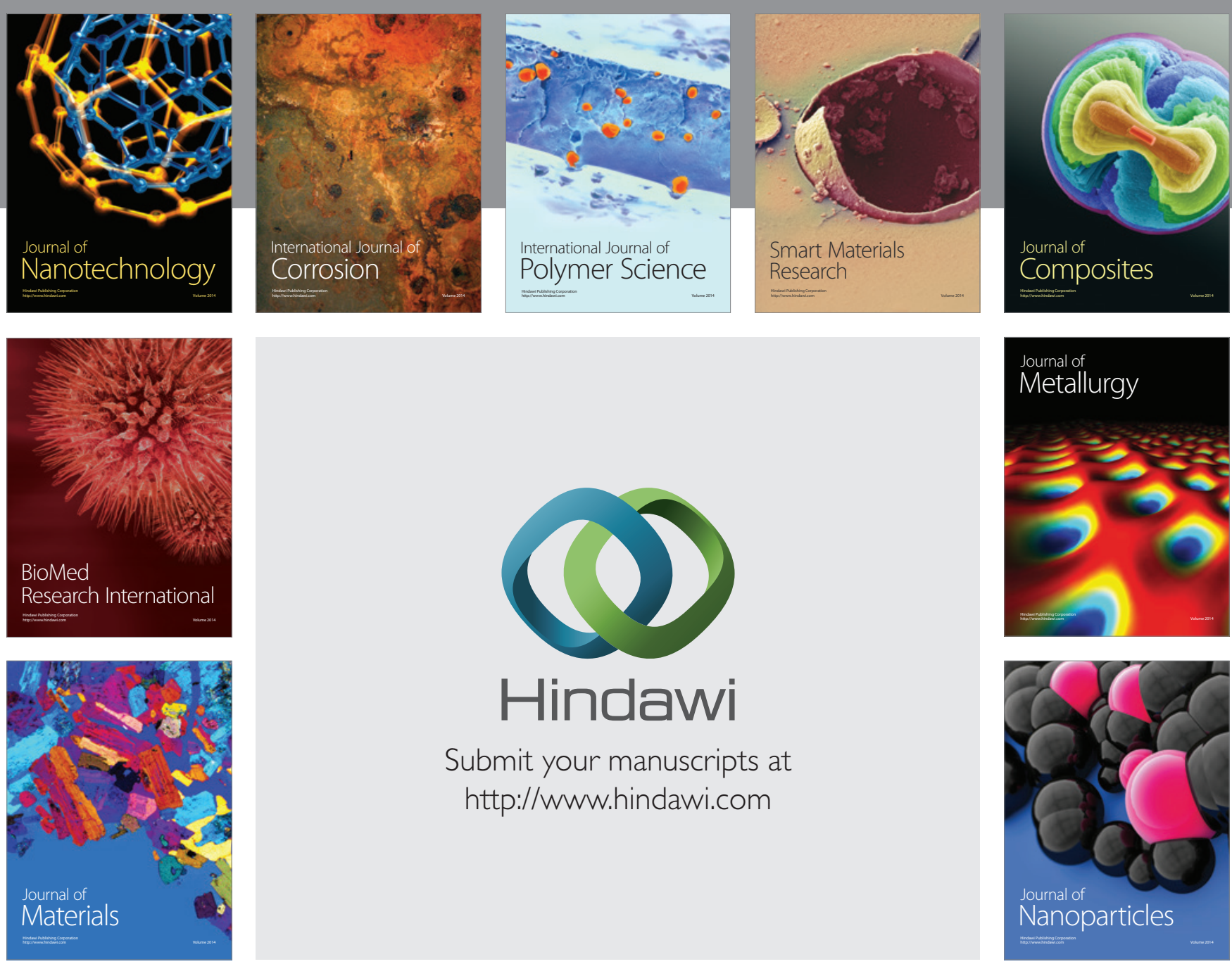

Submit your manuscripts at http://www.hindawi.com
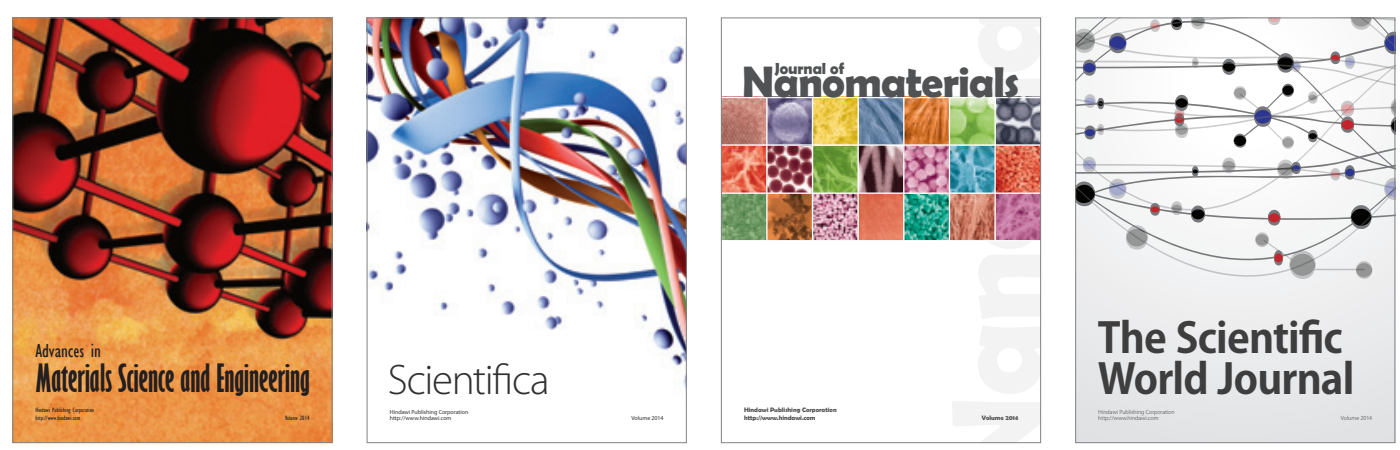

\section{The Scientific World Journal}
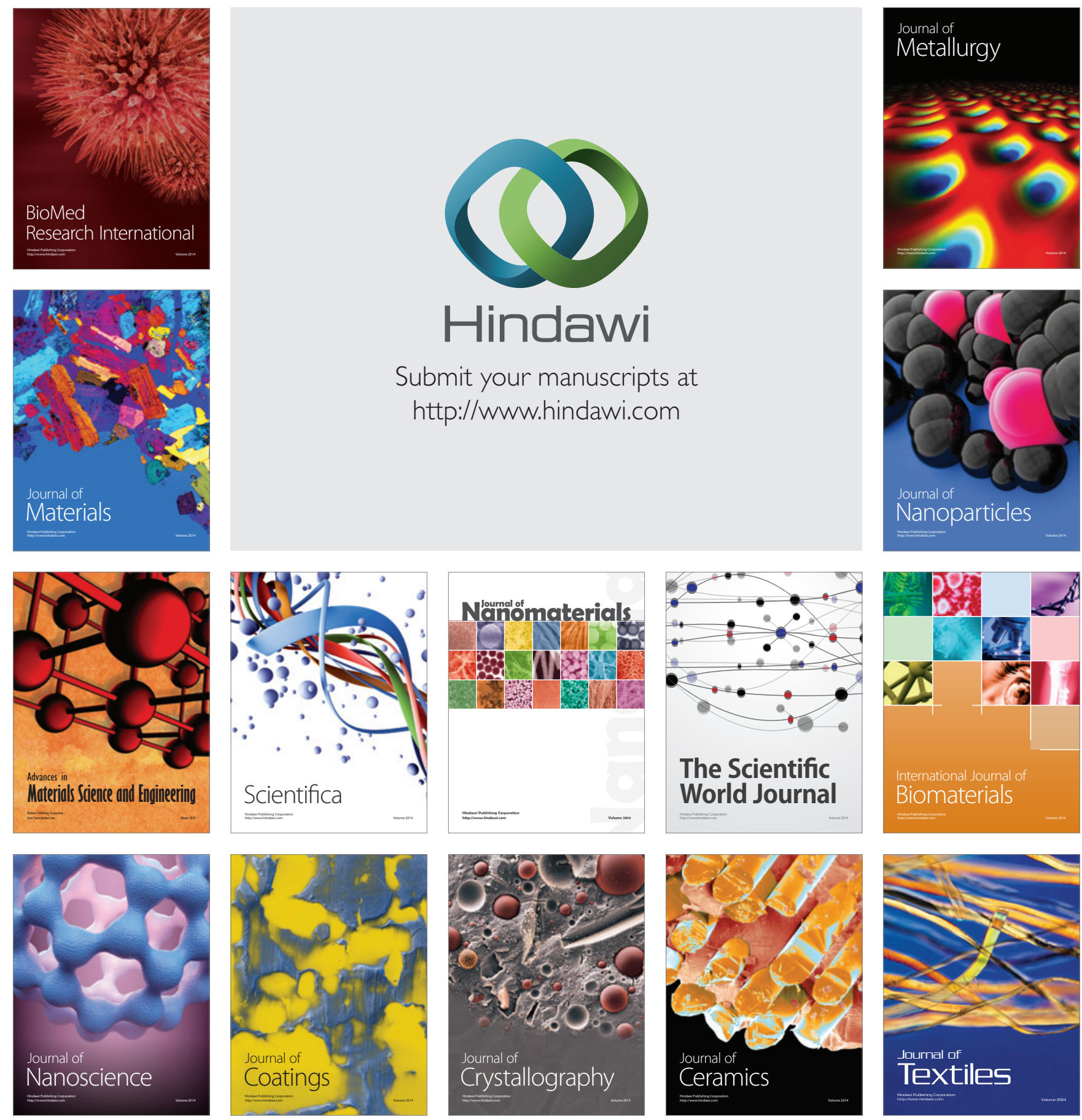